\title{
ncRNAs-mediated upregulation of GTSE1 is involved in the poor prognosis and tumor immune infiltration of Clear Cell Renal Cell Carcinoma
}

kuang xiayu ( $\nabla$ kxy0502@163.com )

shenzhen-shanwei central hospital

cai guogang

shenshan central hospital

Li Canxuan

shenshan central hospital

\section{Research}

Keywords: GTSE1, prognosis ,has-mir-23b-3p, PVT1, non-coding RNA, Tumor immune cell infiltration

Posted Date: June 22nd, 2022

DOl: https://doi.org/10.21203/rs.3.rs-1306942/v2

License: (c) (i) This work is licensed under a Creative Commons Attribution 4.0 International License.

Read Full License 


\section{Abstract}

\section{Aims}

Examine GTSE1 expression and function in renal clear cell carcinoma, as well as the nc-RNA that is upstream of GTSE1.

\section{Methods}

The Cancer Genome Atlas (TCGA) provided clinical and RNA-seq data for ccRCC patients, and the expression of GTSE1, its connection with clinical data, prognosis and tumor immune infiltration, and prediction of upstream noncoding RNAs (nc-RNAs) of GESE1 were analyzed using R language and public tumor databases.

\section{Results}

In cCRCC, GTSE1 is highly expressed and is linked to a high clinical stage and a poor prognosis. In ccRCC, the PVT1 / has-mir-23b-3p / GTSE1 axis has been identified as the most likely upstream ncRNA related route of GTSE1. Furthermore, GTSE1 expression was linked to tumor immune cell infiltration, immune cell biomarker expression, and immunological checkpoint expression.

\section{Conclusions}

Upregulation of GTSE1 by ncRNA is linked to poor prognosis and ccRCC tumor immune infiltration. GTSE1 has the potential to be a useful predictive biomarker as well as a therapeutic target.

\section{Introduce}

The most frequent kind of renal carcinoma is clear cell renal cell carcinoma (ccRCC), which accounts for almost $90 \%$ of all renal cell cancer ${ }^{[1,2]}$. ccRCC is a cancer that is aggressive and has a high risk of metastasis ${ }^{[3,4]}$. Approximately one-third of patients had metastasized at the time of diagnosis, and the remaining one-third may do so in the future ${ }^{[5,6]}$. Because $c$ CRCC is resistant to radiation and chemotherapy ${ }^{[7,8]}$, targeted therapy and immunotherapy are the current therapeutic options. As a result, finding effective treatment targets or promising prognostic indicators for ccRCC is critical.

GTSE1 is mostly found in the cytoplasm and is expressed only during the G2 and S phases of the cell cycle $^{[9]}$, and it is associated with the activity of cytoplasmic tubulin and microtubules during mitosis ${ }^{[10]}$ 10. During mitosis, it is a crucial regulator of chromosomal mobility and spindle integrity ${ }^{[11]}$. The overexpression of GETE1 has been linked to a variety of malignancies. The introduction of p53 into the 
cytoplasm and participation in the control of FoxM1/CCNB1 expression, which is positively linked with tumor recurrence and lymph node infiltration, may mediate the overexpression of GTSE1 in bladder cancer ${ }^{[12]}$. The elevated expression of GTSE1 in hepatocellular carcinoma(HCC) is directly linked to the migration and invasion produced by the regulation of superior dermal epithelial-to-mesenchymal transition (EMT), and may considerably impair chemotherapy efficacy and decrease the survival rate of HCC patients ${ }^{[13]}$. In gastric cancer cells, GTSE1 inhibits p53 apoptotic signal transduction, which can interfere with the impact of cisplatin ${ }^{[14]}$. Furthermore, GTSE1 expression was shown to be considerably higher in triple negative breast cancer and p53 mutant breast cancer cell lines, and was linked to histological grade and a bad prognosis ${ }^{[15]}$. However, detailed study on the expression, prognosis, and regulatory mechanisms of GTSE1 in ccRCC are still lacking. Furthermore, no link between GTSE1 and tumor immune infiltration in ccRCC has been shown.

The administration and mining of a vast number of biological data has become crucial in cancer research due to the rapid development of high-throughput detection technologies and the accumulation of tumor-related data ${ }^{[16]}$. The TCGA, GPS, ICGC, and other databases include a vast amount of publicly available tumor data, which may be used to find new tumor biology markers and therapeutic targets ${ }^{[17-}$ 18]. Using bioinformatics to explore possible treatment targets that will help patients with renal clear cell carcinoma live longer. We investigated the expression and survival of GTSE1 in renal clear cell carcinoma using the public tumor database, as well as the associated regulation of non-coding RNA (ncRNA) and the link between GTSE1 and tumor immunity. Finally, our findings imply that GTSE1 overexpression mediated by ncRNAs is linked to poor prognosis and tumor immune infiltration in patients with ccRCC.

\section{Materials And Methods}

\subsection{Datasets}

The RNA-seq data (HTSeq-FPKM) was obtained from the TCGA database ${ }^{[19]}$ (https://portal.gdc.cancer.gov/). Patients with missing clinicopathological characteristics were eliminated, leaving $530 \mathrm{ccRCC}$ samples and 72 normal samples. For additional investigation, gene expression data from GSE53757 and GSE40435 were also retrieved. GSE53757 [20] is from the GPL570 platform ([HG-U133_plus_2] Affymetrix human genome U133 Plus 2.0 array), while GSE40435 ${ }^{[21]}$ is from the GPL10558 platform (Illumina HumanHT-12 V4.0 expression beadchip). There are 72 and 101 normal samples and 72 and $101 \mathrm{ccRCC}$ samples in each of the two data sets. The R language's "limma" package was used to investigate the differential expression of the target gene in tumor and neighboring normal tissues.

\subsection{Database analysis}


1.2.1 UALCAN ${ }^{[22]}$ (http://ualcan.path.uab.edu/index.html『is a website that provides information on the University of Alabama at It is a network resource that is comprehensive, user-friendly, and interactive. It now offers protein expression analysis choices based on the clinical proteomics tumor analysis Alliance's data (cptac). The ualcan database was used to assess the protein expression level of GTSE1 in ccRCC.

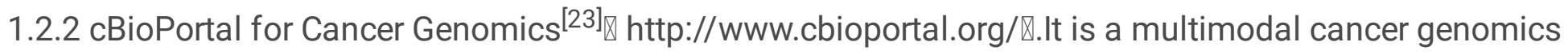
open platform that provides free data on over 5,000 tumor samples from 20 oncology research to far》 contains 5 published tumor data to date, 15 TCGA-provided data. Here, the data set of cCRCC (TCGA, firehose Legacy) was selected for further analysis of GTSE1.

1.2.3 StarBase ${ }^{[24]}$ ( http://starbase.sysu.edu.cn/囚. It's a repository for miRNA, mRNA, IncRNA, and other relevant studies. To predict the miRNA upstream of GTSE1, applications such as PITA, RNA22, miRmap, microT, miRanda, Pictar, and TargetScan are used, and only the projected miRNA that occurs in the above two or more programs is included for further investigation. The expression correlation of miRNA-GTSE1 was further investigated using these predicted miRNAs as potential miRNAs for GTSE1. The final study includes potential miRNAs with a negative association. The target miRNA also predicted the parallel expression of upstream IncRNA, and the analysis included IncRNA with a negative association. Starbase also looked at the levels of miRNA expression in CCRCC and normal controls, the relationship between miRNA and prognosis of ccRCC was also studied on this website.

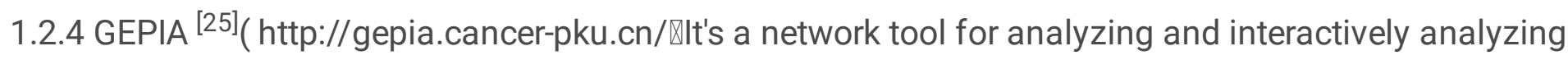
cancer and normal gene expression profiles based on TCGA and genotype tissue expression (GTEX) data. The expression correlation between GTSE1 and immunological checkpoints in ccRCC was assessed using the GEPIA database $R \mid>0.1$ and $P$ value 0.05 were used to define statistically significant selection criterion. This website also looked at the expression of IncRNA in ccRCC and the link between GTSE1, IncRNA, and the prognosis of ccRCC.

1.2.5 TIMER ${ }^{[26]}$ (https://cistrome.shinyapps.io/timer/खlt is a web server that allows for a thorough examination of tumor-infiltrating immune cells. Timer may be used to examine target gene expression in normal and malignant tissues of various malignancies, as well as the relationship between GTSE1 expression and immune cell infiltration or immune checkpoint expression in ccRCC.

\section{3 statistical analysis}

The expression level of GTSE1 in ccRCC tissues was compared to that in normal kidney samples using the Wilcoxon signed rank test. To investigate the relationship between clinicopathological characteristics and GTSE1, the Wilcoxon signed rank test, Kruskal Wallis test, logistic regression, and/or chi square test were utilized. Based on the median value of gene expression, all patients were divided into high and low expression subgroups. The effect of GTSE1 on overall survival of cCRCC patients was assessed using a Kaplan Meier survival analysis and a log rank test. To find independent predictive markers, researchers used univariate Cox regression and multivariate Cox analysis. R 3.6.1 software was used to conduct all 


\section{Result}

\subsection{GTSE1 is substantially expressed in ccRCC}

We discovered that GTSE1 was strongly expressed in most malignancies, including ccRCC, using the timer database (Fig. 1A). TCGA, GSE53757, and GSE40435 transcriptome data revealed that GTSE1 in cCRCC tissues was considerably greater than in nearby normal tissues (Fig. 1B-D). Furthermore, as compared to normal controls, the UALCAN database revealed that GTSE1 was substantially expressed in ccRCC samples at the protein level. (Fig. 1E).

\subsection{Overexpression of GTSE1 was shown to be associated with advanced clinicopathological characteristics.}

We discovered no statistically significant difference in GTSE1 expression in patients with different ages (Fig. 2A),but there were significant difference in different gender and high TNM, histological grade, or clinical stage of cCRCC (Fig. 2B-G). GTSE1 overexpression was found to be significantly associated with pathologic stage (stage III vs stage I OR = 1.95, $\mathrm{P}<0.01$; stage IV vs stage I OR $=4.896, \mathrm{P}<0.001$ ), histologic Grade (Grade 4 vs Grade $1=7.368, \mathrm{P} 0.01$ ); $\mathrm{T}$ classification (T3 vs T1 OR $=2.875, \mathrm{P}<0.001$; T4 vs T1 OR = 2.788, $\mathrm{P}=0.011$ ); $\mathrm{N}$ classification (N1 vs N0 OR=15.126 P<0.001); and M classification (M1 vs $M O O R=2.436, p<0.001$ ) (Table 1 ). The high expression of GTSE1 was connected to histological grade $(p<0.001)$, TNM stage $(p<0.01)$, and pathologic stage $(p<0.001)$, according to chi square test analysis (Table 2). 
Table 1

The relationship between GTSE1 expression and clinicopathological features in ccRCC(Logistic Regression Analysis)

\begin{tabular}{|lllll|}
\hline clinical Parameters & Odds Ratio in GTSE1 & OR.95L & OR.95H & P value \\
\hline age & & & & \\
\hline$<60$ vs $\geq 60$ & 0.941 & 0.669 & 1.323 & 0.728 \\
\hline Gender & & & & \\
\hline Gender (Male vs Female) & 1.442 & 1.008 & 2.067 & 0.046 \\
\hline pathologic_stage & & & & \\
\hline stage2 vs stage1 & 0.928 & 0.51 & 1.659 & 0.803 \\
\hline stage3 vs stage1 & 1.95 & 1.267 & 3.017 & 0.003 \\
\hline stage4 vs stage1 & 4.896 & 2.821 & 8.841 & $<0.001$ \\
\hline histologic grade & & & & \\
\hline Grade2 vs Grade1 & 1.642 & 0.532 & 6.132 & 0.414 \\
\hline Grade3 vs Grade1 & 2.979 & 0.962 & 11.14 & 0.073 \\
\hline Grade4 vs Grade1 & 7.368 & 2.193 & 29.483 & 0.002 \\
\hline T & & & & \\
\hline T2 vs T1 & 1.015 & 2.436 & 7.678 & $<0.001$ \\
\hline T3 vs T1 & 2.875 & 0.588 & 1.732 & 0.957 \\
\hline T4 vs T1 & 14.862 & 1.947 & 4.278 & $<0.001$ \\
\hline M & & 2.788 & 274.722 & 0.011 \\
\hline M1 vs M0 & 4.22 & & & 0.009 \\
\hline N & & & & \\
\hline N1 vs No & & & & \\
\hline
\end{tabular}


Table 2

The relationship between GTSE1 expression and clinicopathological features in ccRCC(Chi-Square Test)

\begin{tabular}{|c|c|c|c|c|c|c|}
\hline characterisics & type & total & $\begin{array}{l}\text { High } \\
\text { expression }\end{array}$ & $\begin{array}{l}\text { Low } \\
\text { expression }\end{array}$ & $\begin{array}{l}\text { Chi } \\
\text { square }\end{array}$ & $\begin{array}{l}p- \\
\text { value }\end{array}$ \\
\hline \multirow[t]{2}{*}{ age } & $>60$ & $266(50.19 \%)$ & 130 (24.5\%) & $134(25.3 \%)$ & 0.07 & 0.794 \\
\hline & $\leq 60$ & $264(49.81 \%)$ & 135 (25.5\%) & 131 (24.7\%) & & \\
\hline \multirow[t]{2}{*}{ gender } & femal & $186(35.09 \%)$ & 104 (19.6\%) & 82 (15.5\%) & 3.65 & 0.056 \\
\hline & male & $344(64.91 \%)$ & $161(30.4 \%)$ & $183(34.5 \%)$ & & \\
\hline \multirow[t]{4}{*}{$\begin{array}{l}\text { histologic } \\
\text { grade }\end{array}$} & G1 & $14(2.68 \%)$ & $10(1.9 \%)$ & $4(0.8 \%)$ & 32.12 & $\begin{array}{l}< \\
0.001\end{array}$ \\
\hline & $\mathrm{G} 2$ & $227(43.49 \%)$ & 137 (26.2\%) & $90(17.2 \%)$ & & \\
\hline & G3 & $206(39.46 \%)$ & $94(18 \%)$ & $112(21.5 \%)$ & & \\
\hline & G4 & 75(14.37\%) & $19(3.6 \%)$ & $56(10.7 \%)$ & & \\
\hline \multirow[t]{4}{*}{$\begin{array}{l}\text { pathologic } \\
\text { stage }\end{array}$} & stage1 & $265(50.28 \%)$ & $158(30 \%)$ & 107 (20.3\%) & 38.72 & $\begin{array}{l}<.001 \\
0.001\end{array}$ \\
\hline & stage2 & $57(10.82 \%)$ & $35(6.6 \%)$ & $22(4.2 \%)$ & & \\
\hline & stage3 & $123(23.34 \%)$ & $53(10.1 \%)$ & 70 (13.3\%) & & \\
\hline & stage4 & $82(15.56 \%)$ & $19(3.6 \%)$ & $63(12 \%)$ & & \\
\hline \multirow[t]{4}{*}{$\mathrm{T}$} & $\mathrm{T} 1$ & $271(51.13 \%)$ & $162(30.6 \%)$ & 109 (20.6\%) & 38.33 & $\begin{array}{l}<.001 \\
0.001\end{array}$ \\
\hline & $\mathrm{T} 2$ & $69(13.02 \%)$ & $41(7.7 \%)$ & $28(5.3 \%)$ & & \\
\hline & T3 & $179(33.77 \%)$ & 61 (11.5\%) & 118 (22.3\%) & & \\
\hline & $\mathrm{T} 4$ & $11(2.08 \%)$ & $1(0.2 \%)$ & $10(1.9 \%)$ & & \\
\hline \multirow[t]{2}{*}{$N$} & NO & $239(93.73 \%)$ & 120 (47.1\%) & 119 (46.7\%) & 9.93 & 0.002 \\
\hline & N1 & $16(6.27 \%)$ & $1(0.4 \%)$ & $15(5.9 \%)$ & & \\
\hline \multirow[t]{2}{*}{ M } & MO & $420(84.34 \%)$ & 227 (45.6\%) & 193 (38.8\%) & 26.11 & $\begin{array}{l}< \\
0.001\end{array}$ \\
\hline & M1 & $78(15.66 \%)$ & 17 (3.4\%) & $61(12.2 \%)$ & & \\
\hline
\end{tabular}

\subsection{Survival outcome and Cox regression analysis}

All patients were split into low and high subgroups based on the median expression value of GTSE1. The predictive value of GTSE1 in patients with CCRCC was evaluated using Kaplan-Meier survival analysis, and a ROC curve was created. The results revealed that patients in the GTSE1 high expression subgroup 
had a worse overall survival rate than those in the low expression subgroup $(P<0.001)$. (Fig. 3A-B). Because of the significant number of patients with missing $\mathrm{N}$ stage and $\mathrm{M}$ stage data in the database, we omitted $\mathrm{N}$ stage patients from the analysis and deleted patients with missing $\mathrm{M}$ stage data to minimize statistical bias.GTSE1 overexpression was strongly related with poor prognosis $(H R=2.027, p 0.001)$, according to the findings of cox regression analysis. Other clinical characteristics, such as age, histological grade, clinical stage, $\mathrm{T}$ stage, and $\mathrm{M}$ stage, were also shown to be associated with a poor prognosis (all $P<0.001)$. GTSE1 expression $(p=0.014)$, age $(P=0.001)$, histological grade $(P=0.013)$, pathologic stage $(P<0.05)$, and distant metastasis $(P<0.001)$ were all shown to be independent variables impacting OS values in multivariate Cox regression analysis (Table 3 ).

Table 3

Univariate and multifactorial cox regression analysis between clinicopathological features and survival in cCRCC(date from TCGA)

\begin{tabular}{|c|c|c|c|c|c|c|c|c|}
\hline \multirow[t]{2}{*}{ Variables } & \multicolumn{4}{|c|}{ Univariate Cox Regression } & \multicolumn{4}{|c|}{ Multivariate Cox Regression } \\
\hline & $\mathrm{HR}$ & HR.95L & HR.95H & p-value & $\mathrm{HR}$ & HR.95L & HR.95H & $\mathrm{p}$-value \\
\hline $\begin{array}{l}\text { age } \\
(>60 \text { vs } \leq 60)\end{array}$ & 1.841 & 1.35 & 2.511 & 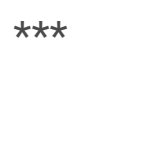 & 1.700 & 1.239 & 2.332 & 0.001 \\
\hline $\begin{array}{l}\text { gender } \\
\text { (male vs female) }\end{array}$ & 1.195 & 0.872 & 1.638 & 0.268 & 1.131 & 0.819 & 1.561 & 0.445 \\
\hline $\begin{array}{l}\text { histologic grade } \\
\text { (G3/G4 vs G1/G2) }\end{array}$ & 2.291 & 1.621 & 3.238 & $\star \star \star$ & 1.592 & 1.103 & 2.298 & 0.013 \\
\hline $\begin{array}{l}\text { pathologic stage } \\
\text { (III/IV vs I/II) }\end{array}$ & 3.226 & 2.329 & 4.468 & 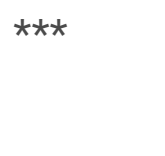 & 2.148 & 1.042 & 4.424 & 0.038 \\
\hline $\begin{array}{l}\mathrm{T} \\
\text { (T3/T4 vs T1/T2) }\end{array}$ & 2.689 & 1.967 & 3.676 & $\star \star \star *$ & 0.868 & 0.462 & 1.630 & 0.648 \\
\hline $\begin{array}{l}\text { M } \\
\text { (M1 vs M0) }\end{array}$ & 3.584 & 2.589 & 4.963 & $\star \star \star$ & 2.217 & 1.497 & 3.281 & 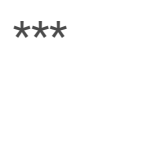 \\
\hline $\begin{array}{l}\text { GTSE1 } \\
\text { (High vs Low) }\end{array}$ & 2.24 & 1.627 & 3.084 & $\star \star \star$ & 1.529 & 1.088 & 2.150 & 0.014 \\
\hline Note: ${ }^{* \star \star} p<0.001$ & & & & & & & & \\
\hline
\end{tabular}

\subsection{MicroRNA prediction}


The role of noncoding RNA (ncRNA) in gene expression control is well understood. To see if any ncRNAs influence GTSE1, we first hypothesized that the upstream miRNA may bind to GTSE1 and then detected 14 miRNAs. miRNA is negatively linked with GTSE1 based on its action mechanism in the control of target gene expression. As a result, an expression correlation analysis was performed. As the Table 4 shown that GTSE1 is strongly negatively linked with hsa-mir-23b-3p, hsa-mir-338-3p, and hsa-mir-532-3p, whereas the other four miRNAs have no statistical expression association (Table 4). Finally, the expression of hsa-mir-23b-3p, hsa-mir-338-3p, and hsa-mir-532-3p in ccRCC was determined, as well as their predictive values (Fig. 4A-E). The same as the number.Only hsa-mir-23b-3p and hsa-mir-532-3p are considerably lowered in ccRCC, and only hsa-mir-23b-3p is up-regulated, suggesting that only hsa-mir$23 b-3 p$ is favorably connected with patient prognosis. All of these data point to hsa-mir-23b-3p as the most likely GTSE1 regulatory miRNA in ccRCC.

Table 4

Correlation analysis between mRNA and miRNA in ccRCC(date from StarBase)

\begin{tabular}{|c|c|c|c|c|}
\hline mRNA & miRNA & $R$ value & p-value & p-star \\
\hline GTSE1 & hsa-miR-23a-3p & 0.124 & 0.0046 & $\star \star \star ~$ \\
\hline GTSE1 & hsa-miR-181a-5p & -0.018 & 0.688 & \\
\hline GTSE1 & hsa-miR-181b-5p & 0.095 & 0.03 & * \\
\hline GTSE1 & hsa-miR-181c-5p & -0.066 & 0.1 & \\
\hline GTSE1 & hsa-miR-23b-3p & -0.238 & 4.08E-08 & $\star \star \star$ \\
\hline GTSE1 & hsa-miR-134-5p & 0.282 & 7.10E-11 & $\star \star \star$ \\
\hline GTSE1 & hsa-miR-150-5p & 0.246 & $1.53 \mathrm{E}-08$ & $\star \star \star$ \\
\hline GTSE1 & hsa-miR-381-3p & 0.224 & $2.81 \mathrm{E}-07$ & $\star \star \star ~$ \\
\hline GTSE1 & hsa-miR-338-3p & -0.051 & 0.025 & * \\
\hline GTSE1 & hsa-miR-181d-5p & 0.082 & 0.06 & \\
\hline GTSE1 & hsa-miR-671-5p & 0.271 & $3.67 \mathrm{E}-10$ & $\star \star \star ~$ \\
\hline GTSE1 & hsa-miR-296-3p & 0.200 & $4.42 \mathrm{E}-06$ & $\star \star \star ~$ \\
\hline GTSE1 & hsa-miR-532-3p & -0.114 & 0.009 & $\star \star \star ~$ \\
\hline GTSE1 & hsa-miR-543 & 0.151 & $5.96 \mathrm{E}-04$ & $\star \star \star$ \\
\hline \multicolumn{5}{|c|}{ Note: ${ }^{*} p<0.05 * *<0.01 * * * p<0.001$} \\
\hline
\end{tabular}




\subsection{Prediction and analysis of upstream IncRNA of hsa-mir- $23 b-3 p$}

Using a SateBase database, the upstream IncRNA of hsa-mir-23b-3p was predicted next. hsa-mir-23b-3p predicted a total of 53 IncRNA. IncRNA may boost mRNA expression by competing with shared miRNA, according to the competing endogenous RNA (ceRNA) theory. As a result, IncRNA and miRNA should have a negative connection, while IncRNA and mRNA should have a positive association. We projected a negative association between InRNA and hsa-mir-23b-3p based on the preceding parameters(Table 5). PVT1 was discovered to be substantially expressed in ccRCC after further investigation(Fig. 5A-B). In terms of expression, survival, and association analyses, PVT1 / has-mir-23b-3p / GTSE1 axis appears to be the most promising upstream Incrna in ccRCC.

Table 5

Correlation analysis between IncRNA and miRNA or IncRNA and miRNA in ccRCC (data from StarBase database)

\begin{tabular}{|llll|}
\hline miRNA/mRNA & LncRNA & R value & p-value \\
\hline hsa-miR-23b-3p & PVT1 & -0.242 & 0.0315 \\
\hline GTSE1 & PVT1 & 0.229293 & $1.02 \mathrm{E}-07$ \\
\hline
\end{tabular}

\subsection{GTSE1 was positively correlated with immune cell infiltration in ccRCC}

We utilized the Timer website to investigate the function of GTSE1 in the immune system. In ccRCC, the infiltration level of CD8 $+T$, CD4 $+T$, neutrophil, and dendritic cells reduced when the copy number of GTSE1 was increased, as shown in Fig. 6a. Further correlation analysis reveals crucial information for the research of GTSE1 function and mechanism. As a result, the relationship between GTSE1 expression and immune cell penetration was investigated. In cCRCC, expression of GTSE1 was substantially linked with all immune cells studied, including B cells, CD8 T cells, CD4 T cells, macrophages, neutrophils, and dendritic cells(Fig. 6B-G).

\subsection{Correlation between the expression of GTSE1 and immune cell biomarkers in cCRCC}

We used the GEPIA database to examine the expression connection between GTSE1 and cCRCC immune cell biomarkers in order to further investigate the role of GTSE1 in tumor immunity. Table 4 shows the relationship between GTSE1 and B cell biomarkers (CD19 and CD79a), CD8 T cell biomarkers (CD8a and CD8b), CD4 T cell biomarkers (CD4), M1 macrophage biomarkers (NOS2, IRF5 and PTGS2), M2 
macrophage biomarkers (CD163, VSIG4 and MS4A4A), neutrophil biomarkers (ITGAM and CCR7). The favorable connection between GTSE1 and immune cell penetration is somewhat supported by these data(Table 6).

Table 6

Correlation analysis between GTSE1 and immune cell biomarkers in CCRCC (date from GEPIA)

\begin{tabular}{|c|c|c|c|c|}
\hline Immune cell & Biomarker & $R$ value & p-value & p-star \\
\hline \multirow[t]{2}{*}{ B cell } & CD19 & 0.051 & 0.085 & \\
\hline & CD79A & 0.28 & 0.047 & * \\
\hline \multirow[t]{2}{*}{$\mathrm{CD} 8+\mathrm{T}$} & CD8A & 0.21 & $1.50 \mathrm{E}-06$ & $\star \star \star$ \\
\hline & $\mathrm{CD} 8 \mathrm{~B}$ & 0.16 & $2.6 \mathrm{E}-04$ & $\star \star \star$ \\
\hline $\mathrm{CD} 4+\mathrm{T}$ & CD4 & 0.22 & $2.4 \mathrm{E}-07$ & $\star \star \star *$ \\
\hline \multirow[t]{3}{*}{ M1 } & Nos2 & -0.017 & 0.7 & \\
\hline & IRF5 & 0.13 & 0.0042 & $\star \star$ \\
\hline & PTGS2 & 0.021 & 0.64 & \\
\hline \multirow[t]{3}{*}{ M2 } & CD163 & 0.27 & $4.8 \mathrm{E}-10$ & $\star \star \star \star$ \\
\hline & VSIG4 & 0.33 & $8.9 \mathrm{E}-15$ & $\star \star \star \star$ \\
\hline & MS4A4A & 0.17 & $6.8 \mathrm{E}-05$ & $\star \star \star$ \\
\hline \multirow[t]{2}{*}{ Neutrophil } & CEACAM8 & 0.024 & 0.58 & \\
\hline & ITGAM & 0.051 & 0.24 & \\
\hline \multirow[t]{8}{*}{ Dendritic cell } & CCR7 & 0.07 & 0.11 & \\
\hline & HLA-DPB1 & 0.055 & 0.21 & \\
\hline & HLA-DQB1 & 0.041 & 0.35 & \\
\hline & HLA-DRA & 0.075 & 0.088 & \\
\hline & HLA-DPA1 & 0.052 & 0.23 & \\
\hline & CD1C & 0.012 & 0.78 & \\
\hline & NRP1 & -0.06 & 0.17 & \\
\hline & ITGAX & 0.18 & $3.5 \mathrm{E}-05$ & $\star \star \star$ \\
\hline Note: ${ }^{*} p<0 . C$ & $\star *<0.01 * *$ & 0.001 & & \\
\hline
\end{tabular}




\subsection{Relationship between GTSE1 and ccRCC immune checkpoint}

The immune checkpoints PDCD1/PD-L1/PD-L2 and CTLA-4 are curcial in tumor immune escape. The relationship between GTSE1 and PDCD1, PD-L1, PD-L2, or CTLA-4 was investigated in light of GTSE1's potential carcinogenic effect in ccRCC. In ccRCC, GTSE1 expression was strongly positively linked with PD1, PD-L2, and CTLA-4 after purity correction, but not with PDCD1. Furthermore, we checked the GTPIA database and discovered that in CCRCC, GTSE1 showed a strong positive connection with PDCD1, PD-L1, and CTLA-4, but no significant correlation with PD-L2(Fig. 7A-H). Other immune cell biomarkers, in addition to neutrophil biomarkers, were found to be more or less favorably linked with GTSE1, indicating a link between GTSE1 and immune cell infiltration.

\section{Discussion}

Advanced ccRCC is still known for having a dismal prognosis ${ }^{[27]}$. The discovery of the molecular mechanism of ccRCC carcinogenesis might lead to the development of efficient treatment targets or the identification of potential prognostic biomarkers. During mitosis, GTSE1 is linked to the activity of cytoplasmic tubulin and microtubules, and the activity of microtubules has a direct impact on the likelihood of chromosomal distribution abnormalities. Increased GTSE1 levels in normal cells result in a greater cell division error rate ${ }^{[28]}$. Indeed, more and more data suggests that GTSE1 expression is linked to malignant prognosis. However, our knowledge of GTSE1 in ccRCC is currently incomplete, and more research is needed.

The involvement of GTSE1 in cCRCC was thoroughly studied in this works. To begin, a review of various public tumor databases revealed that GTSE1 expression in ccRCC rose dramatically. GTSE1 overexpression was linked to a higher histological grade, advanced clinical stage, TNM stage, and a poor prognosis. GTSE1 was also found to be an independent risk factor in individuals with cCRCC in both univariate and multivariate Cox regression analyses. As a result, we concluded that GTSE1 is a significant oncogene in ccRCC.

There is enough evidence that ncRNAs, including as miRNA, Incrna, and circular RNA (circular RNA), communicate with one another via the ceRNA mechanism and play a role in gene regulation. ${ }^{\text {[29-32] }}$. According to this concept, we used the StarBase online tool to forecast 14 miRNAs and then screened two miRNAs, hsa-miR-23b-3p and hsa-miR-532-3p, that were negatively linked with both mRNA and highly expressed in renal paraneoplastic tissues. Although there have been few studies on the role of hsa-mir$23 b-3 p$ and hsa-mir-532-3p in renal tumors, it has been reported that hsa-mir-23b-3p is important in cervical cancer and intrahepatic cholangiocarcinoma ${ }^{[33,34]}$; Hsa-mir-532-3p can also be used as a biological marker for lung adenocarcinoma prognosis ${ }^{[35]}$. Further survival analysis indicates that hsa-mir$23 b-3 p$ is very important in predicting the prognosis of ccRCC, although hsa-mir-532-3p is not. As a result, we believe hsa-mir-23b-3p is the most effective miRNA upstream of GTSE1. 
We also predicted the IncRNAs upstream of hsa-miR-23b-3p, and our study comprised a total of 53 IncRNAs. Only PVT1 was suitable among the final 56 IncRNAs, according to the ceRNA doctrine. The high expression of PVT1 was shown to be substantially linked with the prognosis of ccRCC in a survival study. According to reports, PVT1 plays a part in the incidence and progression of a variety of cancers, influencing tumor proliferation and generating tumor metastasis. For example, PVT1 can promote gallbladder cancer tumor progression through the miR-143 / hK2 axis $^{[36]}$, promote angiogenesis in gastric cancer by activating the STAT3 / VEGFA axis ${ }^{[37]}$, and promote renal cell carcinoma proliferation, invasion, and epithelial mesenchymal transformation by downregulating mir-16-5 $\mathrm{p}^{[38]}$. As a result, we identified PVT1 / hsa-mir-23b-3p / GTSE1 as a possible ccRCC regulatory pathway.

Research has shown that tumor infiltrating immune cells (TIC) play a key role in carcinogenesis and development, as well as influencing cancer patients' treatment outcomes and prognoses ${ }^{[39-41]}$. In ccRCC, GTSE1 was strongly positively connected with B cells, CD8 T cells, CD4 T cells, macrophages, neutrophils, and dendritic cells, according to our findings. In addition to neutrophil biomarkers, gtse1 was shown to be substantially related with biomarkers that penetrate immune cells. Further analysis revealed that M2 macrophages were most closely associated with GTSE1.M2 macrophages are known to enhance tumor cell genesis and metastasis, as well as suppress T cell-mediated antitumor immune responses, accelerate tumor angiogenesis, and worsen prognosis ${ }^{[41-42]}$. Residing in the above findings, tumor immune infiltration may partially explain the oncogenic role and prognosis of GTSE1 in ccRCC.

Clear cell renal carcinoma targeted treatment and immunotherapy are essential therapeutic strategies for advanced renal cell carcinoma, and immune checkpoint expression is a key connection impacting the

therapeutic efficacy ${ }^{[43]}$. As a result, we looked into the connection between GTSE1 and the immunological checkpoint. The findings revealed that elevated GTSE1 expression in CCRCC was connected to PDCD1, PD-L1, PD-L2, or CTLA-4, implying that targeted GTSE1 can improve immunotherapy effectiveness in ccRCC.

Finally, high GTSE1 expression is associated with poor prognosis, tumor immune cell infiltration, and immune detection sites in ccRCC, and the PVT1/hsa-miR-23b-3p/GTSE1 axis is an important regulatory mechanism for ccRCC. The above pathway could be a promising therapeutic target for cCRCC. Furthermore, we must not overlook the flaws in tumor databases and the constraints of retrospective research; we must also confirm our findings with more fundamental tests and large-scale clinical trials.

\section{Conclusions}

The expression of PVT1/hsa-mir-23b-3p/GTSE1 axis gene is highly correlated with the clinical characteristics of ccRCC, which can predict its prognosis and guide clinical individualized treatment. Our study provides important evidence for detecting the role of GTESE1 in renal cell carcinoma in the future.

\section{Abbreviations}




\begin{tabular}{ll} 
TCGA & The Cancer Genome Atlas \\
\hline GEO & Gene Expression Omnibus \\
\hline ccRCC & Clear Cell Renal Cell Carcinoma \\
\hline nc-RNAs & noncoding RNAs \\
\hline HCC & hepatocellular carcinoma \\
\hline EMT & epithelial-to-mesenchymal transition \\
\hline GTEX & TCGA and genotype tissue expression \\
\hline ceRNA & competing endogenous RNA \\
\hline TIC & tumor infiltrating immune cells
\end{tabular}

\section{Declarations}

\section{Ethical approval and Consent to participate}

This article does not contain any studies with human participants or animals performed by any of the authors.

\section{Consent for publication}

All authors consent to the publication of this study.

\section{Availability of supporting data}

The RNA-sequencing data and corresponding clinical information were downloaded from The Cancer Genome Atlas (TCGA) database (https://portal.gdc.cancer.gov/) and Gene Expression Omnibus (GEO) (https://www.ncbi.nlm.nih.gov/geo/) database

\section{Competing interests}

No competing interests.

\section{Funding}

No funding was received.

\section{Authors' contributions}

I certify that each co-author listed on page 1 participated sufficiently in the work to take responsibility for the content, and that all those who qualify are listed. Authorship credit should be based on (a) substantial contributions to the conception or design of the work; or the acquisition, analysis, or interpretation of data for the work; AND (b) drafting the work or revising it critically for important intellectual content; AND (c) 
final approval of the version to be published; AND (d) agreement to be accountable for all aspects of the work in ensuring that questions related to the accuracy or integrity of any part of the work are appropriately investigated and resolved.

Acknowledgements[We thank the reviewers for their valuable comments

\section{Contributor Information}

Kuang Xiayu email: kxy0502@163.com

Cai Guogang: email: 1248351751@qq.com

Li Canxuan: email:china20170713@163.com

\section{References}

1. Hsieh JJ, Purdue MP, Signoretti S, et al. Renal cell carcinoma. Nat Rev Dis Primers. 2017;3:17009. doi:10.1038/nrdp.2017.9. Published 2017 Mar 9.

2. Gray RE, Harris GT. Renal Cell Carcinoma: Diagnosis and Management. Am Fam P. 2019 Feb 1;99(3):179-184. Erratum in: Am Fam Physician. 2019 Jun 15;99(12):732. PMID: 30702258.

3. Xie Y, Chen L, Ma X, Li H, Gu L, Gao Y, Fan Y, Zhang Y, Zhang X. Prognostic and clinicopathological role of high Ki-67 expression in patients with renal cell carcinoma: a systematic review and metaanalysis. Sci Rep. 2017;7:44281.

4. Girgis $H$, Masui $O$, White NM, Scorilas A, Rotondo F, Seivwright A, Gabril M, Filter ER, Girgis AH, Bjarnason GA, Jewett MA, Evans A, Al-Haddad S, Siu KM, Yousef GM. Lactate dehydrogenase A is a potential prognostic marker in clear cell renal cell carcinoma. Mol Cancer. 2014;13:101.

5. He H, Wang L, Zhou W, Zhang Z, Wang L, Xu S, Wang D, Dong J, Tang C, Tang H, Yi X, Ge J. MicroRNA expression profiling in clear cell renal cell carcinoma: identification and functional validation of key miRNAs. PLoS ONE. 2015;10:e0125672.

6. Xia Y, Zhang QF, Zhen Q, Zhao Y, Liu NJ, Li T, Hao YN, Zhang Y, Luo CL, Wu XH. Negatively regulation of tumor-infiltrating NK cell in clear cell renal cell carcinoma patients through the exosomal pathway. Oncotarget. 2017;8:37783-95.

7. Makhov P, Joshi S, Ghatalia P, Kutikov A, Uzzo RG, Kolenko VM. Resistance to Systemic Therapies in Clear Cell Renal Cell Carcinoma: Mechanisms and Management Strategies. Mol Cancer Ther. 2018 Jul;17(7):1355-1364. doi: 10.1158/1535-7163.MCT-17-1299. PMID: 29967214; PMCID: PMC6034114.

8. Vaziri SA, Grabowski DR, Hill J, Rybicki LR, Burk R, Bukowski RM, Ganapathi MK, Ganapathi R. Inhibition of proteasome activity by bortezomib in renal cancer cells is p53 dependent and VHL independent. Anticancer Res. 2009;29:2961-9. 
9. Monte M, Collavin L, Lazarevic D, Utrera R, Dragani TA, Schneider C. Cloning, chromosome mapping and functional characterization of a human homologue of murine Gtse-1(B99) gene. Gene. 2000;254:229-36. doi:10.1016/S0378-1119(00)00260-2.

10. Monte M, Benetti R, Buscemi G, Sandy P, Del Sal G, Schneider C. The cell cycle-regulated protein human GTSE-1 controls DNA damage-induced apoptosis by affecting p53 function. J Biol Chem. 2003;278:30356-64. doi:10.1074/jbc.M302902200.

11. Tipton AR, Wren JD, Daum JR, Siefert JC, Gorbsky GJ. GTSE1 regulates spindle microtubule dynamics to control Aurora B kinase and Kif4A chromokinesin on chromosome arms. J Cell Biol. 2017;216:3117-32. doi:10.1083/jcb.201610012.

12. Liu A, Zeng S, Lu X, Xiong Q, Xue Y, Tong L, Xu W, Sun Y, Zhang Z, Xu C. Overexpression of $G 2$ and $S$ phase-expressed-1 contributes to cell proliferation, migration, and invasion via regulating p53/FoxM1/CCNB1 pathway and predicts poor prognosis in bladder cancer. Int J Biol Macromol. 2019 Feb 15;123:322-334. doi: 10.1016/j.ijbiomac.2018.11.032. Epub 2018 Nov 8. PMID: 30414902.

13. Wu X, Wang H, Lian Y, Chen L, Gu L, Wang J, Huang Y, Deng M, Gao Z, Huang Y. GTSE1 promotes cell migration and invasion by regulating EMT in hepatocellular carcinoma and is associated with poor prognosis. Sci Rep. 2017 Jul 11;7(1):5129. doi: 10.1038/s41598-017-05311-2. PMID: 28698581; PMCID: PMC5505986.

14. Subhash VV, Tan SH, Tan WL, Yeo MS, Xie C, Wong FY, Kiat ZY, Lim R, Yong WP. GTSE1 expression represses apoptotic signaling and confers cisplatin resistance in gastric cancer cells. BMC Cancer. 2015;15:550. doi:10.1186/s12885-015-1550-0.

15. Lin F, Xie YJ, Zhang XK, Huang TJ, Xu HF, Mei Y, Liang H, Hu H, Lin ST, Luo FF, Lang YH, Peng LX, Qian CN, Huang BJ. GTSE1 is involved in breast cancer progression in p53 mutation-dependent manner. J Exp Clin Cancer Res. 2019 Apr 8;38(1):152. doi: 10.1186/s13046-019-1157-4. PMID: 30961661; PMCID: PMC6454633.

16. Hutter C, Zenklusen JC. The cancer genome atlas: creating lasting value beyond its data. Cell. 2018;173(2):283-5. doi:10.1016/j.cell.2018.03.042.

17. Zhang J, Bajari R, Andric D. The international cancer genome consortium data portal. Nat Biotechnol. 2019;37(4):367-9. doi:10.1038/s41587-019-0055-9.

18. von Roemeling CA, Radisky DC, Marlow LA, et al. Neuronal pentraxin 2 supports clear cell renal cell carcinoma by activating the AMPA-selective glutamate receptor-4. Cancer Res. 2014;74(17):4796810. doi:10.1158/0008-5472.CAN-14-0210.

19. Blum A, Wang P, Zenklusen JC. SnapShot. TCGA-Analyzed Tumors. Cell. 2018 Apr 5;173(2):530. doi: 10.1016/j.cell.2018.03.059. PMID: 29625059.

20. von Roemeling CA, Radisky DC, Marlow LA, et al. Neuronal pentraxin 2 supports clear cell renal cell carcinoma by activating the AMPA-selective glutamate receptor-4. Cancer Res. 2014;74(17):4796810. doi:10.1158/0008-5472.CAN-14-0210. 
21. Wozniak MB, Le Calvez-Kelm F, Abedi-Ardekani B, Byrnes G, Durand G, Carreira C, Michelon J, Janout V, Holcatova I, Foretova L, Brisuda A, Lesueur F, McKay J, Brennan P, Scelo G. Integrative genomewide gene expression profiling of clear cell renal cell carcinoma in Czech Republic and in the United States. PLoS ONE. 2013;8(3):e57886. doi:10.1371/journal.pone.0057886. Epub 2013 Mar 5. PMID: 23526956; PMCID: PMC3589490.

22. Chandrashekar DS, Bashel B, Balasubramanya SAH, Creighton CJ, Rodriguez IP, Chakravarthi BVSK, Varambally S. UALCAN: A portal for facilitating tumor subgroup gene expression and survival analyses. Neoplasia. 2017 Aug;19(8):649-658. doi: 10.1016/j.neo.2017.05.002 [PMID:28732212].

23. Gao J, Aksoy BA, Dogrusoz U, Dresdner G, Gross B, Sumer SO, Sun Y, Jacobsen A, Sinha R, Larsson E, Cerami E, Sander C, Schultz N. Integrative analysis of complex cancer genomics and clinical profiles using the cBioPortal. Sci Signal. 2013 Apr 2;6(269):pl1. doi: 10.1126/scisignal.2004088. PMID: $23550210 ;$ PMCID: PMC4160307.

24. starBase v2.0: decoding miRNA-ceRNA, miRNA-ncRNA and protein-RNA interaction networks from large-scale CLIP-Seq data.Li JH, Liu S, Zhou H. Qu LH* and Yang JH*Nucleic Acids Res. 2014;42:D92-7.

25. Tang Z, Li C, Kang B, Gao G, Li C, Zhang Z. GEPIA: a web server for cancer and normal gene expression profiling and interactive analyses. Nucleic Acids Res. 2017;45(W1):W98-102. [PMC free article] [PubMed] [Google Scholar].

26. Li T, Fu J, Zeng Z, Cohen D, Li J, Chen Q, Li B, Liu XS. TIMER2.0 for analysis of tumor-infiltrating immune cells. Nucleic Acids Res. 2020 Jul 2;48(W1):W509-W514. doi: 10.1093/nar/gkaa407. PMID: 32442275; PMCID: PMC7319575.

27. Pierorazio PM, Johnson MH, Patel HD, et al. Management of Renal Masses and Localized Renal Cancer: Systematic Review and Meta-Analysis. J Urol. 2016;196(4):989-99. doi:10.1016/j.juro.2016.04.081.

28. Lai W, Zhu W, Li X, Han Y, Wang Y, Leng Q, Li M, Wen X. GTSE1 promotes prostate cancer cell proliferation via the SP1/FOXM1 signaling pathway. Lab Invest. 2021 May;101(5):554-563. doi: 10.1038/s41374-020-00510-4. Epub 2020 Dec 16. PMID: 33328578.

29. Chan JJ, Tay Y. Noncoding RNA:RNA Regulatory Networks in Cancer. Int J Mol Sci. 2018 Apr 27;19(5):1310. doi: 10.3390/ijms19051310. PMID: $29702599 ;$ PMCID: PMC5983611.

30. Fiscon G, Conte F, Farina L, Paci P. Network-Based Approaches to Explore Complex Biological Systems towards Network Medicine. Genes (Basel). 2018 Aug 31;9(9):437. doi: 10.3390/genes9090437. PMID: 30200360; PMCID: PMC6162385.

31. Yamamura S, Imai-Sumida M, Tanaka Y, Dahiya R. Interaction and cross-talk between non-coding RNAs. Cell Mol Life Sci. 2018 Feb;75(3):467-84. doi:10.1007/s00018-017-2626-6. Epub 2017 Aug 24. PMID: 28840253; PMCID: PMC5765200.

32. Razavi ZS, Tajiknia V, Majidi S, Ghandali M, Mirzaei HR, Rahimian N, Hamblin MR, Mirzaei H. Gynecologic cancers and non-coding RNAs: Epigenetic regulators with emerging roles. Crit Rev Oncol Hematol. 2021;157:103192. 
33. Hu J, Sun Z, Hu K, Tang M, Sun S, Fang Y, Yu H, Zhang Y. [Over-expression of Hsa-miR-23b-3p suppresses proliferation, migration, invasion and epithelial-mesenchymal transition of human cervical cancer CasKi cells]. Xi Bao Yu Fen Zi Mian Yi Xue Za Zhi. 2020 Nov;36(11):983-989. Chinese. PMID: 33210592.

34. Lei GL, Li Z, Li YY, Hong ZX, Wang S, Bai ZF, Sun F, Yan J, Yu LX, Yang PH, Yang ZY. Long noncoding RNA FAM66C promotes tumor progression and glycolysis in intrahepatic cholangiocarcinoma by regulating hsa-miR-23b-3p/KCND2 axis. Environ Toxicol. 2021 Nov;36(11):2322-32. doi:10.1002/tox.23346. Epub 2021 Aug 21. PMID: 34418280.

35. Wang Z, Pei H, Liang H, Zhang Q, Wei L, Shi D, Chen Y, Zhang J. Construction and Analysis of a circRNA-Mediated ceRNA Network in Lung Adenocarcinoma. Onco Targets Ther. 2021 Jun 8;14:3659-3669. doi: 10.2147/OTT.S305030. PMID: 34135596; PMCID: PMC8197624.

36. Chen J, Yu Y, Li H, Hu Q, Chen X, He Y, Xue C, Ren F, Ren Z, Li J, Liu L, Duan Z, Cui G, Sun R. Long noncoding RNA PVT1 promotes tumor progression by regulating the miR-143/HK2 axis in gallbladder cancer. Mol Cancer. 2019 Mar 2;18(1):33. doi: 10.1186/s12943-019-0947-9. PMID: 30825877; PMCID: PMC6397746.

37. Zhao J, Du P, Cui P, Qin Y, Hu C, Wu J, Zhou Z, Zhang W, Qin L, Huang G. LncRNA PVT1 promotes angiogenesis via activating the STAT3/VEGFA axis in gastric cancer. Oncogene. 2018 Jul;37(30):4094-4109. doi: 10.1038/s41388-018-0250-z. Epub 2018 Apr 30. PMID: 29706652.

38. Ren Y, Huang W, Weng G, Cui P, Liang H, Li Y. LncRNA PVT1 promotes proliferation, invasion and epithelial-mesenchymal transition of renal cell carcinoma cells through downregulation of miR-165p. Onco Targets Ther. 2019 Apr 5;12:2563-2575. doi: 10.2147/OTT.S190239.

39. Waniczek D, Lorenc Z, Śnietura M, Wesecki M, Kopec A, Muc-Wierzgoń M. Tumor-Associated Macrophages and Regulatory T Cells Infiltration and the Clinical Outcome in Colorectal Cancer. Arch Immunol Ther Exp (Warsz ). 2017;65:445-54.

40. Petitprez F, Meylan M, de Reyniès A, Sautès-Fridman C, Fridman WH. The Tumor Microenvironment in the Response to Immune Checkpoint Blockade Therapies. Front Immunol. 2020 May 7;11:784. doi: 10.3389/fimmu.2020.00784. PMID: 32457745; PMCID: PMC7221158.

41. Jarosz-Biej M, Smolarczyk R, Cichoń T, Kułach N. Tumor Microenvironment as A "Game Changer" in Cancer Radiotherapy. Int J Mol Sci. 2019 Jun 29;20(13):3212. doi: 10.3390/ijms20133212. PMID: 31261963; PMCID: PMC6650939.

42. Plesca I, Tunger A, Müller $L$, et al. Characteristics of Tumor-Infiltrating Lymphocytes Prior to and During Immune Checkpoint Inhibitor Therapy. Front Immunol. 2020;11:364. doi:10.3389/fimmu.2020.00364. Published 2020 Mar 4.

43. Postow MA, Sidlow R, Hellmann MD. Immune-Related Adverse Events Associated with Immune Checkpoint Blockade. N Engl J Med. 2018 Jan 11;378(2):158-168. doi: 10.1056/NEJMra1703481. PMID: 29320654.

\section{Figures}


A

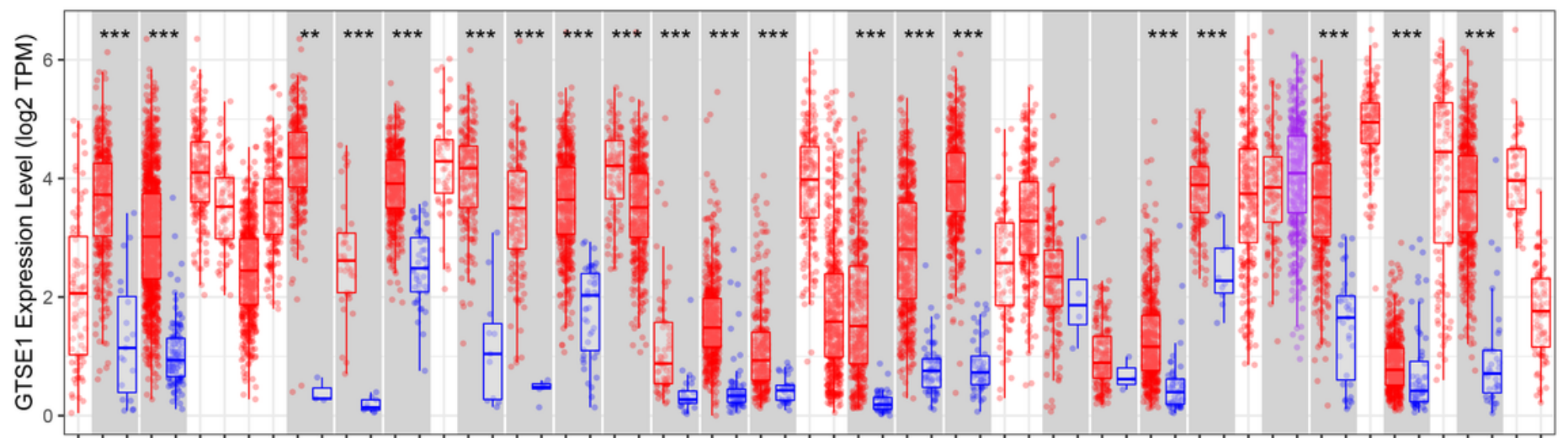

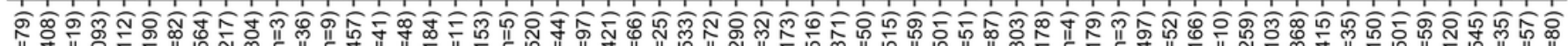

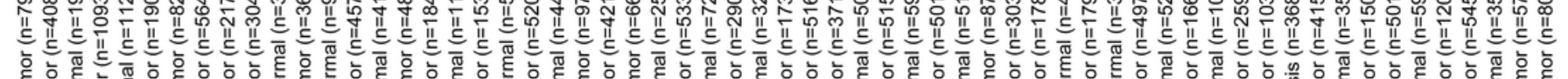

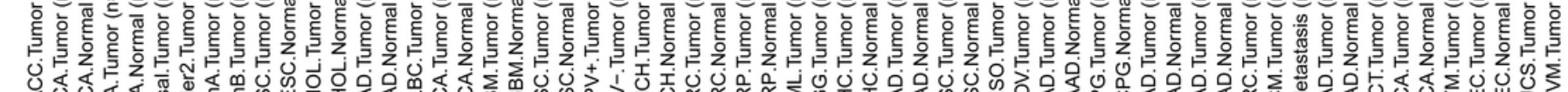

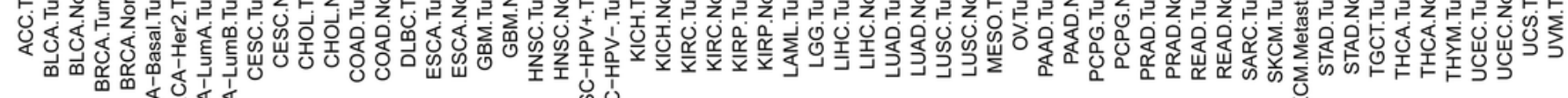

$\mathrm{B}$

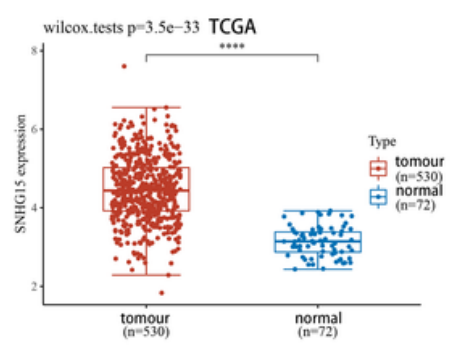

垔空

$\mathrm{C}$

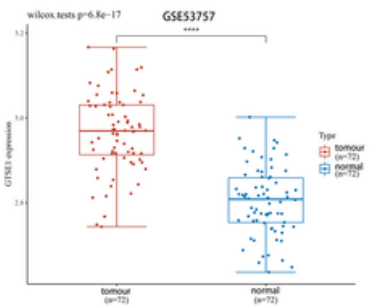

$\mathrm{D}$

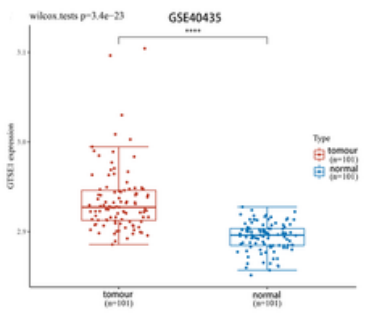

采

$\mathrm{E}$

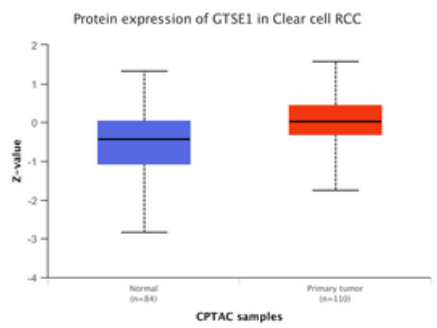

Figure 1

GTSE1 were highly expressed in ccRCC samples. (A) GTSE1 expression levels in various tumor types from TCGA database were determined by TIMER; (B-D) GTSE1 was substantially expressed in ccRCC tissues, according to data from TCGA, GSE53757, and GSE40435; (E) Based on the UALCAN database, we found that the expression levels of GTSE1 protein were higher in ccRCC samples than that in normal renal tissues 
A

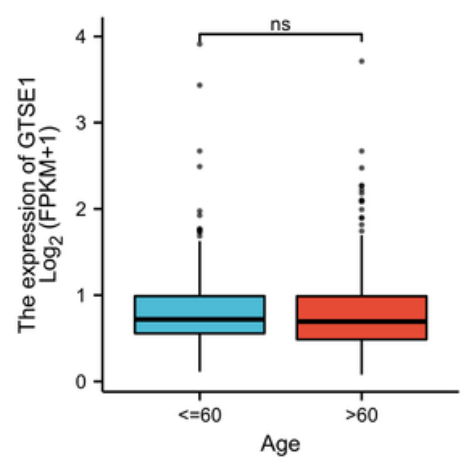

$\mathrm{B}$

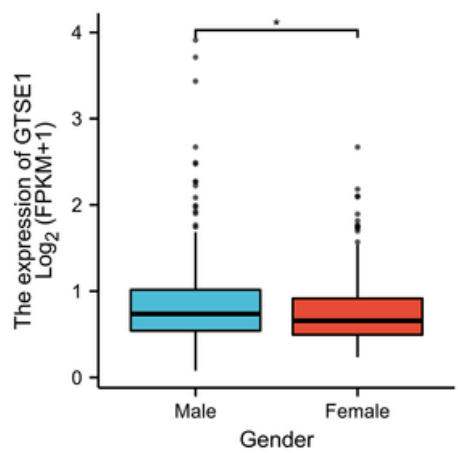

$\mathrm{E}$

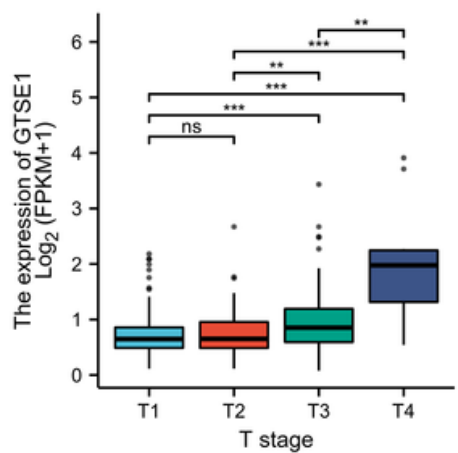

G
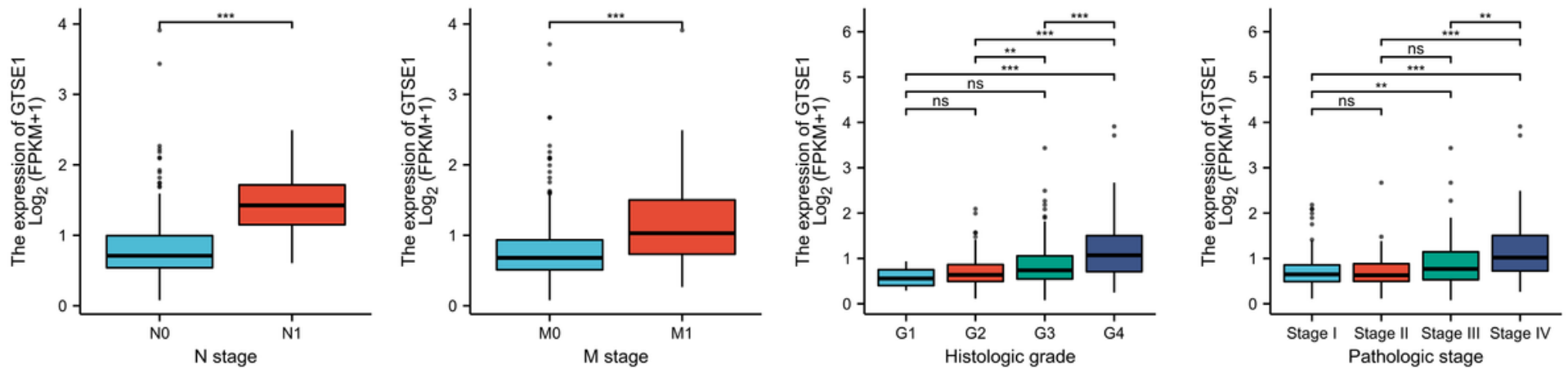

Figure 2

The relationship between GTSE1 expression and clinicopathological features in ccRCC (A) Age; (B) Gender; (C) T Stage; (D) N Stage ; (E) M Stage; (F) Histologic grade; (G) Pathologic Stage. Notes: * $\mathrm{p}<$ $0.05, * \star p<0.01, * * * p<0.001$.

\section{Figure 3}

Survival analysis and Cox regression analysis (A) Patients with elevated GESE1 expression had a worse overall survival rate according to a Kaplan-Meier survival study; (B) ROC curves and AUC of GTSE1 at different times in patients with ccRCC 
A

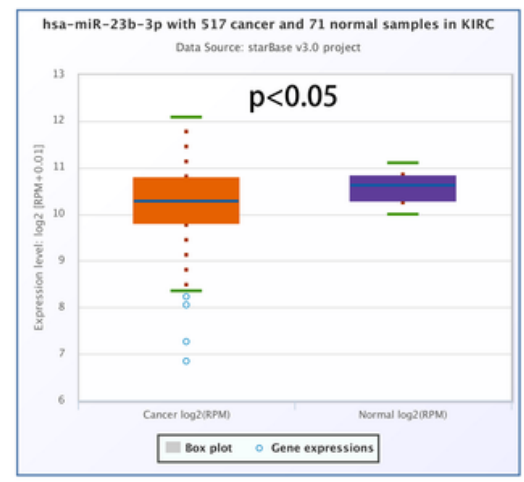

$\mathrm{B}$

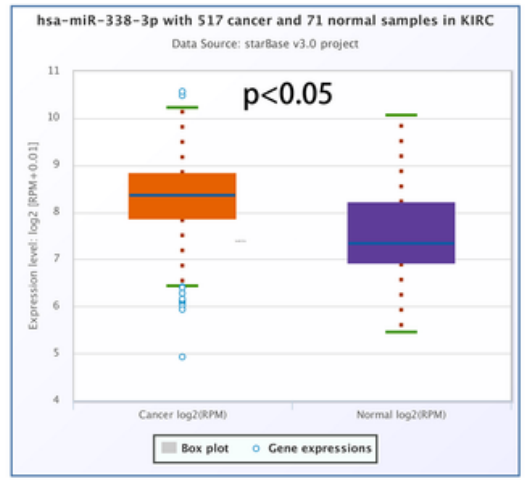

$\mathrm{C}$

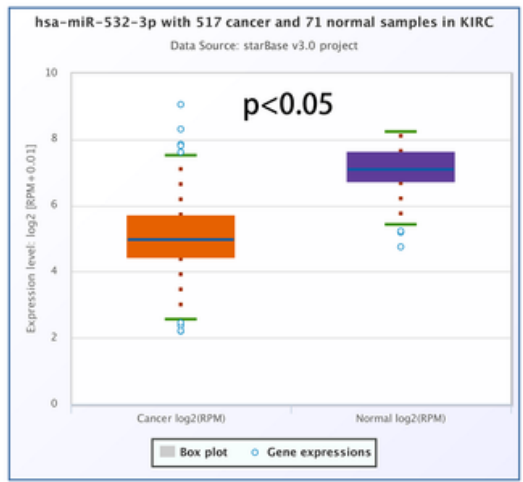

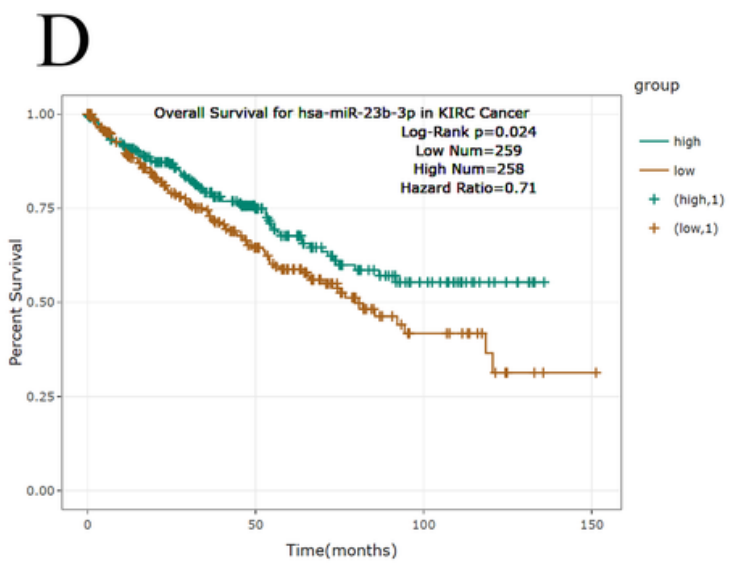

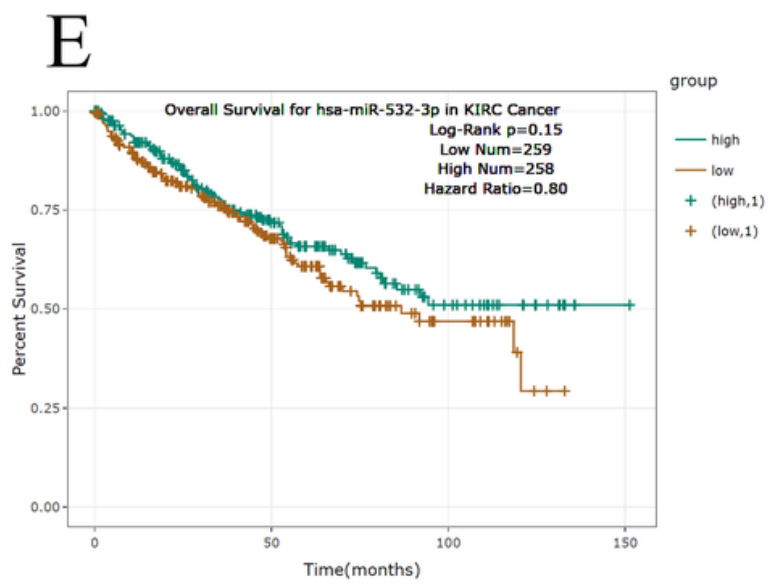

Figure 4

Expression and survival analysis of different miRNAs in ccRCC (A-C) The expression of target miRNA in cCRCC and control normal samples determined by StarBase database. (D-E) StarBase evaluated the predictive value of hsa-miR-23b-3p and hsa-miR-532b-3p in ccRCC.

\section{Figure 5}

Expression and survival analysis of IncRNA(PVT1) in ccRCC (A) Analysis of PVT1 expression in ccRCC based on GEPIA website (B) Analysis of PVT1 survival analysis in cCRCC based on GEPIA website. 


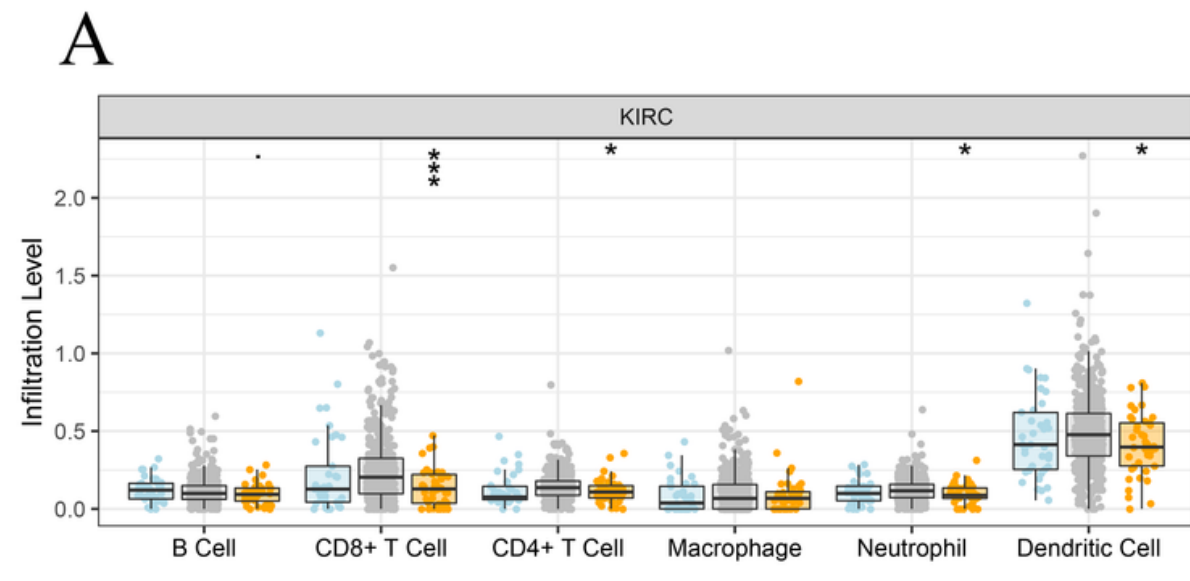

Copy Number

审 Arm-level Deletion

审 Diploid/Normal

审 Arm-level Gain

\section{B}

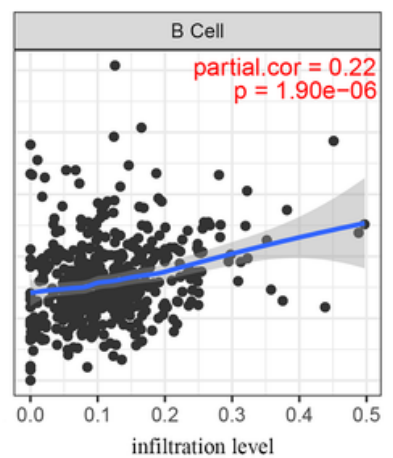

E

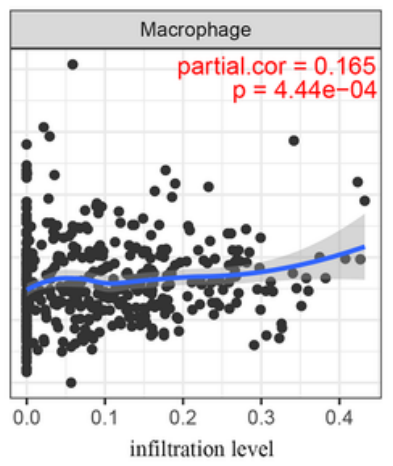

C

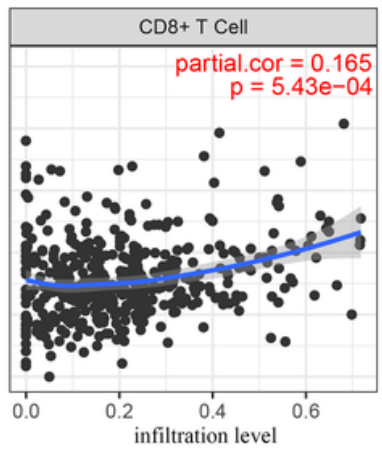

F

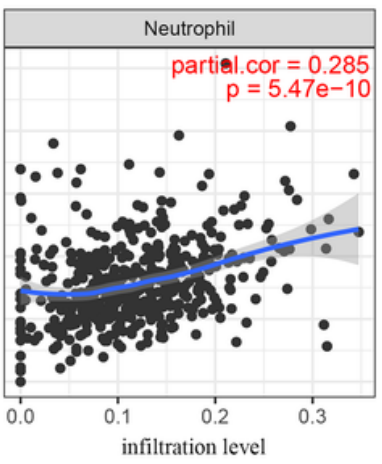

D

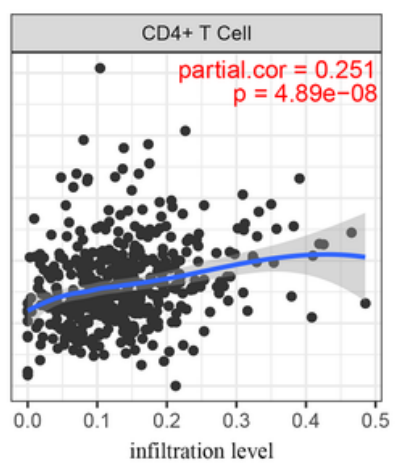

G

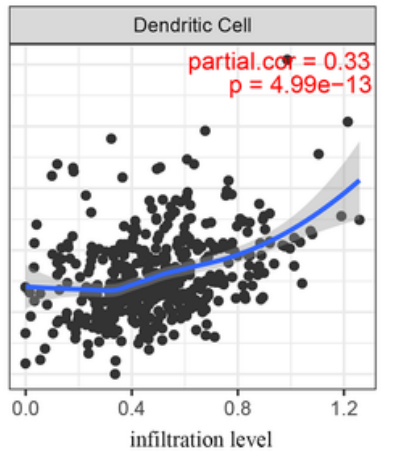

\section{Figure 6}

The relationship of immune cell infiltration with GTSE1 level in ccRCC base on TIMER website (A) Varying immune cells infiltrate at varied levels in ccRCC with different copy numbers of GTSE1. (B-G) The correlation of GTSE1 expression level with B cell, CD8 T cell, CD4 T cell, Macrophage, Neutrophil, or Dendritic cell infiltration level in ccRCC. Notes: ${ }^{\star} p<0.05,{ }^{\star *} p<0.01,{ }^{\star \star *} p<0.001$. 

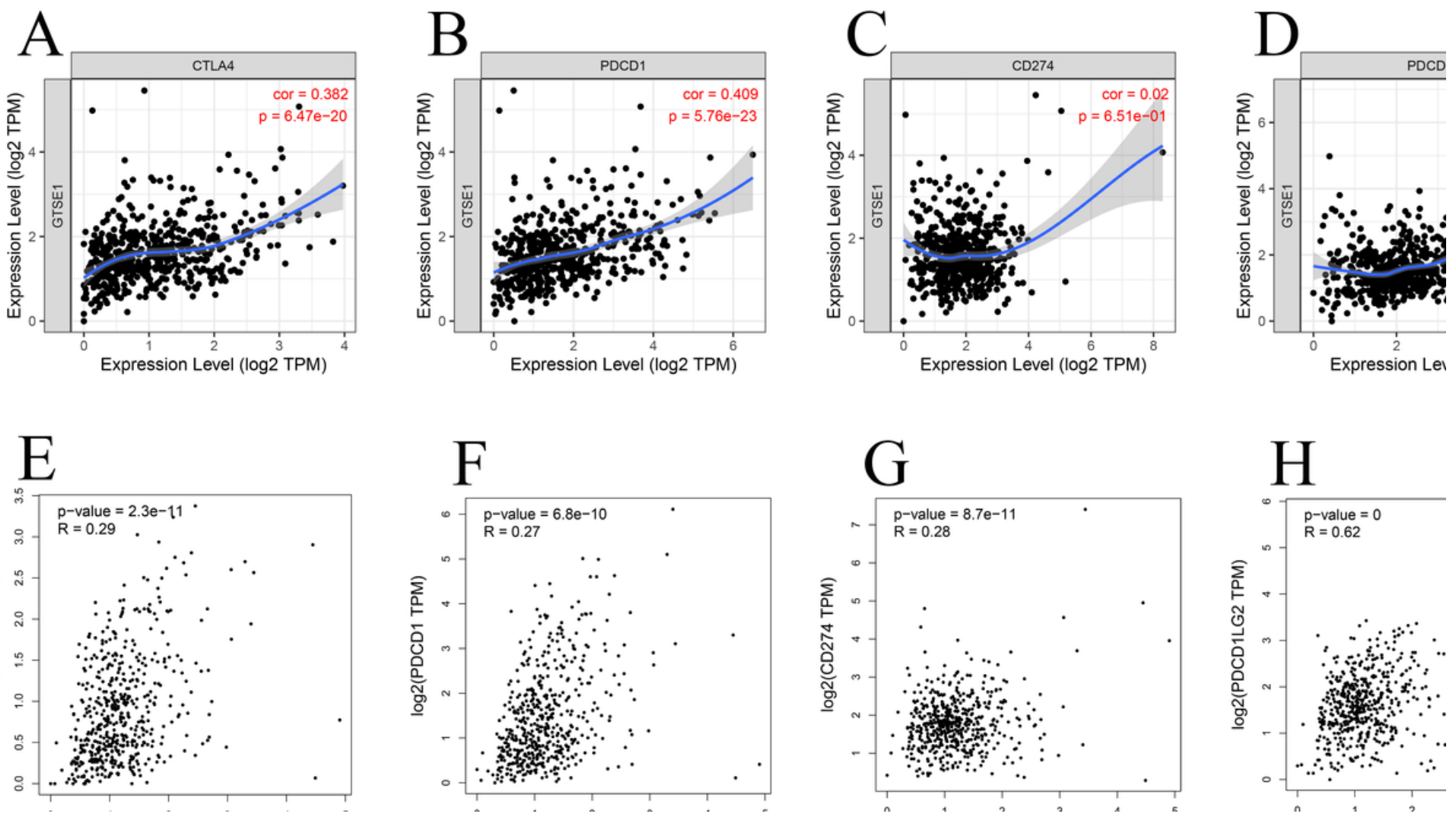

Figure 7

Correlation analysis of GTSE1 expression and immune checkpoints in ccRCC (A-D) CTLA4,PDCD1,PDL1,PD-L2 and GTSE1 correlation analysis in ccRCC determined by TIMER website (E-H) CTLA4,PDCD1,PD-L1,PD-L2 and GTSE1 correlation analysis in ccRCC determined by GEPIA website. 